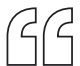

The growth

of Sputnik in

the giant viral

factories of

mamavirus

significantly

impaired the

production of

normal mamavirus

virions.

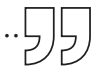

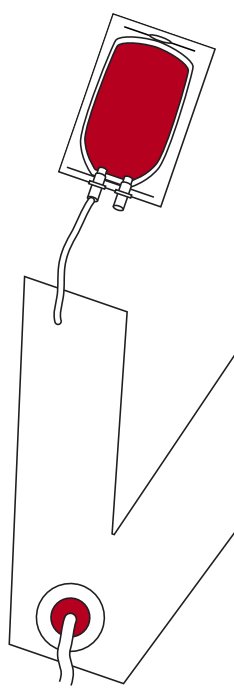

VIROLOGY

\title{
A virus gets a virus
}

Raoult and co-workers previously challenged the field of virology by identifying a virus that was so large its size questioned the definition of a virus. Now, publishing in Nature, La Scola, Desnues, Raoult and colleagues describe a new strain of the giant mimivirus that has its own viral infection.

The previously identified Acanthamoeba polyphaga mimivirus, which only grows in amoebae, was isolated from a cooling tower in Bradford, United Kingdom. Mimivirus was visible under an optical microscope and became the largest known virus. Now, following inoculation of $A$. polyphaga with water from a cooling tower in Paris, France, La Scola et al. have identified a new strain of mimivirus that is even larger. They named this new strain mamavirus and its main features closely resemble those of mimivirus, including the formation of giant viral factories in host cells. Fascinatingly, however, they found that mamavirus was not alone in the infected A. polyphaga cells - also present was an unknown, small icosahedral virus that was $50 \mathrm{~nm}$ in size, which they named Sputnik.

Sputnik did not grow when inoculated into Acanthamoeba castellanii. However, it did grow in A. castellanii cells that were coinfected with mamavirus, and its effect on mamavirus was deleterious. The growth of Sputnik in the giant viral factories of mamavirus significantly impaired the production of normal mamavirus virions, which indicates that Sputnik is a genuine parasite of mamavirus. Although satellite viruses of animals or plants also need a helper virus to replicate, this is the first time that a virus has been identified that uses the viral factory of another virus to propagate at the expense of the helper virus. The authors have proposed that Sputnik is a 'virophage' owing to its functional analogy with bacteriophages.

The Sputnik genome is an 18,343 bp circular double-stranded DNA with 21 predicted protein-encoding genes. Of these, eight encode proteins with homologues. Three of these eight genes seem to be derived

from mimivirus or mamavirus, which indicates that this virophage participates in gene-transfer processes and mediates lateral gene transfer between giant viruses.

The other five genes encode proteins that are homologues of an archaeal virus integrase, a predicted primase-helicase, a packaging ATPase, a distant homologue of bacterial insertion sequence transposase DNA-binding subunit and a zincribbon protein. Interestingly, the closest homologues of the last four proteins were detected in the Global Ocean Sampling Expedition, which indicates that Sputnik is the first member of a new family of viruses that is abundantly represented in marine metagenomic sequences.

\section{Rachel Smallridge}

ORIGINAL RESEARCH PAPER La Scola, B. et al. The virophage as a unique parasite of the giant mimivirus. Nature 6 Aug 2008 (doi:10.1038/ nature07218)

FURTHER READING Raoult, D. \& Forterre, P.

Redefining viruses: lessons from Mimivirus.

Nature Rev. Microbiol. 6, 315-319 (2008) 